\title{
Características Tomográficas de la Anatomía Laríngea Normal en pacientes que acuden al Servicio de Medicina en Imágenes del Hospital de Clínicas San Lorenzo, Paraguay 2020
}

\author{
Tomographic Characteristics of Normal Laryngeal Anatomy in patients \\ attending the Medical Imaging Service of the Hospital de Clínicas San Lorenzo, \\ Paraguay 2020
}

\author{
Juan Francisco Santacruz-Agüero [1, Carlos Enrique Mena-Canata $\mathbb{D}^{1}$
}

${ }^{1}$ Universidad Nacional de Asunción, Facultad de Ciencias Médicas, San Lorenzo, Paraguay.

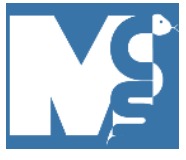

Recibido: $21 / 06 / 2021$

Revisado: 02/08/2021

Aceptado:20/08/2021

\section{Autor correspondiente}

Juan Francisco Santacruz Agüero. Universidad Nacional de Asunción juansanta92js@gmail.com

\section{Conflictos de interés}

Los autores declaran no poseer conflictos de interés.

\section{Fuente de financiación}

Los autores no recibieron apoyo financiero para la investigación, autoría y/o publicación de este artículo.

Este artículo es publicado bajo una licencia de Creative Commons Reconocimiento 4.0 Internacional.

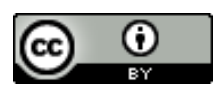

\section{RESUMEN}

Introducción: La tomografía computarizada es el estudio Gold standard para complementar a la endoscopia en el estudio de patologías laríngeas. Por lo tanto, se debe tener conocimiento de la anatomía normal de la laringe. Metodología: Se realizó un estudio observacional descriptivo, de corte transversal retrospectivo. Se recolecto los datos a través del análisis tomográfico, se analizaron 26 tomografías de pacientes que acudieron al Servicio de Imágenes del Hospital de Clínicas sin patología laríngea. Las variables investigadas fueron características tomográficas como: Calcificación cartílagos laríngeos, Altura cartílago tiroideo, Distancia tiro-aritenoidea, Distancia interaritenoidea, Altura y diámetro del espacio preepiglótico. Resultados: Se lograron identificar cada una de las características anatómicas radiológicas buscadas. Además, se realizaron las mediciones correspondientes, detallando el promedio de cada una de las mediciones y clasificándolo por sexo. La altura de cartílago tiroideo la media en hombres fue $28.05 \pm 1.2 \mathrm{~mm}$ y en mujeres fue $27.8 \pm 0.92 \mathrm{~mm}$, para la distancia Tiro-aritenoidea en hombres fue $12.3 \pm 1.3$ y en mujeres $10.97 \pm 0.95 \mathrm{~mm}$, para el diámetro transversal del cartílago tiroideo en hombres fue $16.63 \pm 1.58 \mathrm{~mm}$ y en mujeres $17.96 \pm 1.11 \mathrm{~mm}$. Conclusión: Los promedios de las mediciones realizadas fueron mayor en hombres excepto en el diámetro transversal del cartílago tiroideo, La prevalencia de calcificación de cartílagos laríngeos fue del $54 \%$ de los pacientes estudiados..

Palabras clave: anatomía laríngea normal; tomografía computarizada; calcificación cartílagos laríngeos; altura cartílago tiroideo; distancia tiro-aritenoidea; interaritenoidea; espacio preepiglótico.

\section{ABSTRACT}

Introduction: Computed tomography is the Gold standard study to complement endoscopy in the study of laryngeal pathologies. Therefore, knowledge of the normal anatomy of the larynx is required. Methodology: A retrospective cross-sectional descriptive observational study was carried out. Data were collected through tomographic analysis, 26 tomographies of patients who attended the Imaging Service of the Hospital de Clínicas without laryngeal pathology were analyzed. The variables investigated were tomographic characteristics such as: laryngeal cartilage calcification, thyroid cartilage height, thyro-arytenoid distance, interarytenoid distance, height and diameter of the pre-epiglottic space. Results: Each of the radiological anatomical characteristics sought were identified. In addition, the corresponding measurements were taken, detailing the average of each of the measurements and classifying them by sex. The average thyroid cartilage height in men was $28.05 \pm 1.2 \mathrm{~mm}$ and in women was $27.8 \pm 0.92 \mathrm{~mm}$, for the thyro-arytenoid distance in men was $12.3 \pm 1.3$ and in women $10.97 \pm 0.95 \mathrm{~mm}$, for the transverse diameter of the thyroid cartilage in men was $16.63 \pm 1.58 \mathrm{~mm}$ and in women $17.96 \pm 1.11 \mathrm{~mm}$. Conclusions: The averages of the measurements taken were higher in men except for the transverse diameter of the thyroid cartilage. The prevalence of laryngeal cartilage calcification was $54 \%$ of the patients studied

Keywords: normal laryngeal anatomy, computed tomography, laryngeal cartilage calcification, thyroid cartilage height, thyro-arytenoid, interarytenoid distance, preepiglottic space.. 


\section{INTRODUCCIÓN}

La laringe es un órgano fundamental por sus diversas funciones. En cuanto a su exploración, la laringoscopia indirecta constituye un examen básico y es indispensable para precisar la movilidad de la laringe, pero gracias a la tomografía computarizada (TC) la exploración radiológica es cada vez más precisa, para complementar la endoscopia completa el análisis de la laringe (1). La introducción de la TC supuso una innovación importante en las imágenes laríngeas porque la TC permite diferenciar diferencias mucho más pequeñas en densidades radiológicas que las técnicas convencionales $y$, por tanto, la TC puede ayudar a aclarar la patología submucosa (2).

La TC ha sido favorecida durante mucho tiempo por los radiólogos para la obtención de imágenes laríngeas porque la rapidez de la adquisición de imágenes minimiza los artefactos de respiración y deglución que pueden hacer que las imágenes de RM sean ilegibles (3-4).

Los resultados mostraron que la TC da una evaluación exacta de la anatomía laríngea y la afectación tumoral, particularmente para mostrar los cartílagos laríngeos, la epiglotis y el espacio preepiglótico, la infiltración paracordal y la afectación de las comisuras anterior y posterior (5).

Entre las ventajas que presenta la TC sobre otras modalidades de examen son: representación de las relaciones estructurales con la laringe en el plano axial, definición más precisa de las estructuras cartilaginosas que podrían estar afectadas por tumores en comisura anterior o en supraglotis y la capacidad de ajustar las técnicas de exploración para diferenciar distintos tipos de tejidos (por ej.: musculo) (6).

El conocimiento de la anatomía y de la radioanatomía normal de la laringe es la base indispensable para una interpretación adecuada de las imágenes patológicas de la región laríngea (7).

El objetivo de este trabajo fue determinar las características de estructuras anatómicas de la laringe normal identificables a la tomografía computarizada en pacientes que acuden al Servicio de Medicina en Imágenes del Hospital de Clínicas en el periodo entre marzo y noviembre de 2020. Las características anatómicas estudiadas fueron la altura del cartílago tiroideo por sexo, distancia entre cartílagos tiroideoaritenoideo por sexo, diámetro transversal de tiroides por sexo, la distancia interaritenoidea por sexo, la altura y diámetro mayor del espacio preepiglótico por sexo. Además, la frecuencia de calcificación de cartílagos laríngeos.

\section{METODOLOGÍA}

Se realizó un estudio observacional descriptivo, de corte transversal retrospectivo. Se recolecto los datos a través del análisis tomográfico mediante el programa Radiant dicom viewer de los pacientes con tomografía computarizada cervical que acudieron al Servicio de Medicina en Imágenes del Hospital de Clínicas San Lorenzo Paraguay en el periodo entre marzo y noviembre de 2020, que cumplieron con los criterios de inclusión y exclusión.

El muestreo fue no probabilístico por conveniencia. Las variables investigadas fueron características sociodemográficas de los pacientes, Calcificación cartílagos laríngeos, Altura cartílago tiroideo, Distancia tiro-aritenoidea, Distancia interaritenoidea, Altura y diámetro del espacio preepiglótico.

En cuanto a las referencias utilizadas para las mediciones fueron las siguientes; para la altura de cartílago tiroides se midió entre ambos extremos del asta mayor y menor del cartílago tiroides, para la distancia tiro-aritenoidea fue medido desde la apófisis vocal del aritenoides a la comisura anterior, con respecto al diámetro transversal del cartílago tiroides, se midió en un punto equidistante sobre la distancia tiro-aritenoidea, para la distancia interaritenoidea se midió entre las apófisis musculares, para el espacio preepiglotico se midió la altura y el diámetro de dicho espacio.

Los criterios de inclusión tomados fueron: pacientes que cuenten con tomografías computarizadas de región cervical (cortes $\leq 2,5 \mathrm{~mm}$; axiales) y pacientes con edad mayor o igual a 18 años. Como criterio de exclusión pacientes con diagnóstico de patologías o alteraciones estructurales o funcionales de la laringe.

Para el procesamiento y análisis de los datos se empleó el programa Excel (Microsoft 2013) con sus herramientas. Se llevó a cabo un análisis descriptivo de todas las variables. Se respetaron los principios de beneficencia, no maleficencia, justicia y autonomía.

\section{RESULTADOS}

Se analizaron 26 tomografías de pacientes que acudieron al Servicio de Imágenes del Hospital de Clínicas. De los cuales el $53 \%$ eran hombres, con una media de edad de 40,07 $\pm 12,15$ años. Las mediciones realizadas se describen en la Tabla 1 . En cuanto a la media de altura de cartílago tiroides que se midió entre ambos extremos del asta mayor y menor del cartílago tiroides en mujeres fue de $27,8 \mathrm{~mm}$ en hombres 28,05 $\mathrm{mm}$.

La media distancia tiro-aritenoidea fue de $12,3 \mathrm{~mm}$ en hombres y $10,97 \mathrm{~mm}$ en mujeres, que fue medido 
desde la apófisis vocal del aritenoides a la comisura anterior. Respecto al diámetro transversal del cartílago tiroides, se midió en un punto equidistante sobre la distancia tiro-aritenoidea, la media en mujeres fue de17,96 $\mathrm{mm}$ y en hombres $16,63 \mathrm{~mm}$. La distancia interaritenoidea en mujeres fue de $8,9 \mathrm{~mm}$ y $9,5 \mathrm{~mm}$ en hombres. En cuanto al espacio preepiglótico en hombres la media de altura fue de $14,64 \mathrm{~mm}$ y el diámetro de $8,8 \mathrm{~mm}$, en mujeres la altura media del espacio preepiglótico fue $11,59 \mathrm{~mm}$ y de su diámetro fue de 7,3 mm. La calcificación de los cartílagos laríngeos se presentó en 14 de los pacientes estudiados, siendo la media de edad de estos de 50,14 años, el cartílago tiroideo se encontró calcificado en todos estos, el cricoides en 13 de estos pacientes y el aritenoides en 8 de estos pacientes.

TABLA 1. PROMEDIO DE MEDICIONES MORFORADIOLÓGICAS REALIZADAS EN TOMOGRAFÍAS.

\begin{tabular}{|c|c|c|}
\hline MEDIDA & \multicolumn{2}{|c|}{ PROMEDIO (mm) } \\
\hline Altura de cartílago tiroideo & Hombres: $28.05 \pm 1.2$ & Mujeres: $27.8 \pm 0.92$ \\
\hline Distancia Tiro-aritenoidea & Hombres: $12.3 \pm 1.3$ & Mujeres: $10.97 \pm 0.95$ \\
\hline Diámetro transversal del cartílago tiroideo & Hombres: $16.63 \pm 1.58$ & Mujeres:17.96 \pm 1.11 \\
\hline Distancia interaritenoidea & Hombres: $9.5 \pm 0.88$ & Mujeres: $8.9 \pm 0.75$ \\
\hline Altura del espacio preepiglótico & Hombres: $14.64 \pm 0.92$ & Mujeres: $11.59 \pm 1.23$ \\
\hline Diámetro del espacio preepiglótico & Hombres: $8.83 \pm 1.22$ & Mujeres: $7.32 \pm 1.54$ \\
\hline
\end{tabular}

\section{DISCUSIÓN}

Conocer las características tomográficas de la laringe normal tiene importancia en el momento de reconocer alteraciones en la misma, ya que la tomografía computarizada constituye el método de elección para el estudio de este órgano, por ejemplo, en patologías malignas, como en el diagnóstico del carcinoma de laringe.

La bibliografía nos muestra muy pocos trabajos de estudios de las características tomográficas de la laringe, esto fue una limitación al momento de realizar el tamaño muestral. Nuestros resultados muestran variaciones con respecto a los reportados por Montoya 1989 (8), con respecto a la altura del cartílago tiroideo y distancia tiroideo-aritenoidea, esto puede deberse a que no se especifica en dicho estudio las referencias anatómicas utilizadas para la medición. Con respecto al diámetro transversal del cartílago tiroideo, concuerda con Silverman 1982 (9) que refiere el ángulo más agudo entre las láminas del cartílago tiroideo para los hombres por lo tanto menor diámetro transversal que en las mujeres, esto se manifiesta en la diferencia en cuanto a la característica de la voz en hombres y mujeres, siendo más agudo en mujeres y más grave en los hombres.

En cuanto a la calcificación de cartílagos laríngeos concuerda con la literatura con respecto a la edad de presentación y siendo los más prevalentes el cartílago tiroides y cricoides $(10,11)$. Hay que destacar también la amplia variabilidad en la longitud de los pliegues vocales y diferencias en la simetría, lo que puede evidenciar la existencia de variaciones relacionadas con el tamaño promedio corporal y de las características raciales.

\section{CONTRIBUCIÓN DE LOS AUTORES}

JFSA: concepción y diseño de trabajo, recolección y obtención de resultados, análisis e interpretación de datos, redacción del manuscrito y aprobación de la versión final. CEMC: concepción y diseño de trabajo, recolección y obtención de resultados, análisis e interpretación de datos, redacción del manuscrito y aprobación de la versión final

\section{REFERENCIAS}

1. Instituto Nacional de Estadísticas, Departamento de Extranjería y Migración. Estimación de personas extranjeras residentes habituales en Chile al 31 de Diciembre de 2019. 2020.

2. Chevalier D, Dubrulle F, Vilette B. Anatomía descriptiva, endoscópica y radiológica de la laringe. EMC Otorrinolaringología. $\quad 2001 ; \quad 30(4): 1-14$. https://doi.org/10.1016/S1632-3475(01)71987-X

3. Castelijns J, Van den Brekel M, Hermans R. Imaging of the Larynx. Seminars in Roentgenology. 2000; 35(1): 31-4. https://doi.org/10.1016/S0037-198X(00)80030-9

4. Glastonbury, Christine. Non-Oncologic Imaging of the Larynx. Otolaryngol Clin N Am. 2008; 41: 139-156. https://doi.org/10.1016/i.otc.2007.10.004

5. Suojanen J, Mukherji S, Wippold F. Spiral CT of the Larynx. AJNR. 1994; 15: 1579-1582. URL.

6. Gregor R, Michaels L. Computed Tomography of the Larynx: a clinical and pathologic study. H\&NS 1981; 3(1): 284-296. https://doi.org/10.1002/hed.2890030405

7. Bergman A, Neiman H. Computed Tomography of the 
Larynx. Laringoscope. 1979; 89: 812-817.

https://doi.org/10.1288/00005537-197905000-00019

8. Céruse P, Ltaief-Boudrigua A, Buiret G, Cosmidis A, Tringali S. Anatomía descriptiva, endoscópica y radiológica de la $\begin{array}{llll}\text { laringe. } & \text { EMC. 2012; 41(3): }\end{array}$ https://doi.org/10.1016/S1632-3475(12)62682-4

9. Peñarrocha Montoya L. La Tomografía Computarizada en el estudio de cáncer de laringe y sus metástasis regionales. Tesis Doctorado. Universidad Complutense de Madrid; 1989.

10. Silverman $\mathrm{P}$, Korobkin $\mathrm{M}$. High Resolution Computed Tomography of the normal larynx. AJR. 1983; 140(5):875879. Silverman P, Korobkin M. High Resolution Computed Tomography of the normal larynx. AJR

11. Poch Broto J, Pérez Carretero M. Otorrinolaringología y patología cervicofacial. 1a ed. Madrid: Médica Panamericana; 2006.

12. Katsantonis G, Archer C, Rosenblum B, Yeager V, Friedman W. The Degree to Which Accuracy of Preoperative Staging of Laryngeal Carcinoma has been Enhanced by Computed Tomography. OtolaryngologyHead and Neck Surgery. 1986; 95(1):52-62. https://doi.org/10.1177\%2F019459988609500112 